\title{
Constant-Mean-Curvature Surfaces with Singularities in Minkowski 3-Space
}

\author{
Yuhei Umeda
}

\section{CONTENTS}

1. Introduction

2. Preliminaries

3. Constant-Mean-Curvature Surfaces with Singularities

4. Types of Singularities of Extended CMC Surfaces

5. Examples

References
2000 AMS Subject Classification: Primary 53A10;

Secondary 53C43, 53C50

Keywords: CMC surface, Gauss map, cuspidal edge, swallowtail, singularity
We shall investigate spacelike constant-mean-curvature surfaces with singularities in Minkowski 3-space. Recently, Kokubu, Rossman, Saji, Umehara, and Yamada gave useful criteria for cuspidal edges and swallowtails. Applying their criteria, we define a new class of generalized constant-mean-curvature surface and give some examples with cuspidal edges and swallowtails.

\section{INTRODUCTION}

It is well known that the only complete spacelike maximal (mean-curvature-zero) surface in Minkowski 3-space $\mathbb{L}^{3}$ is the plane. Estudillo and Romero introduced a notion of generalized maximal surfaces in terms of a holomorphic (Weierstrass-type) representation [Estudillo and Romero 92].

A generalized maximal surface without branch points is called a maxface, which was introduced in [Umehara and Yamada 06]; see Figure 1 for typical examples. Umehara and Yamada showed that maxfaces have several interesting global geometric properties. A generic classification of singularities on maxfaces is given in [Fujimori et al. 08].

In contrast to the maximal case, there are many complete spacelike nonzero constant-mean-curvature surfaces (CMC surfaces, for short) in $\mathbb{L}^{3}$ (see Figure 2). For example, the hyperboloid and the Lorentz cylinder are typical. Moreover, Akutagawa constructed many examples by constructing harmonic maps from the hyperbolic plane to itself [Akutagawa 94]. Furthermore, H. Wang constructed complete surfaces (without singularities) by solving the sinh-Gordon equation [Wang 91] (see Figure 3). On the other hand, there are natural examples of CMC surfaces with singularities, including surfaces of revolution. Since singularities of such surfaces are considered wave-front singularities in 3-dimensional space, it is expected that generic wave-front singularities, that 


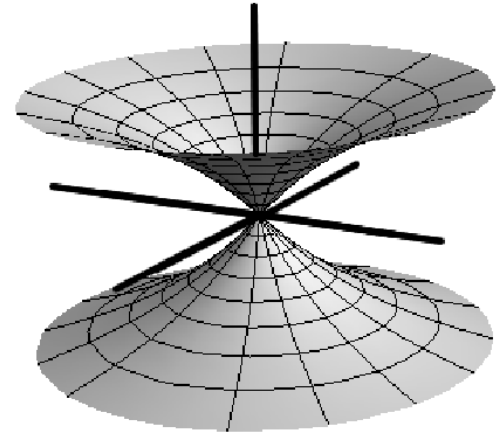

Lorentz catenoid

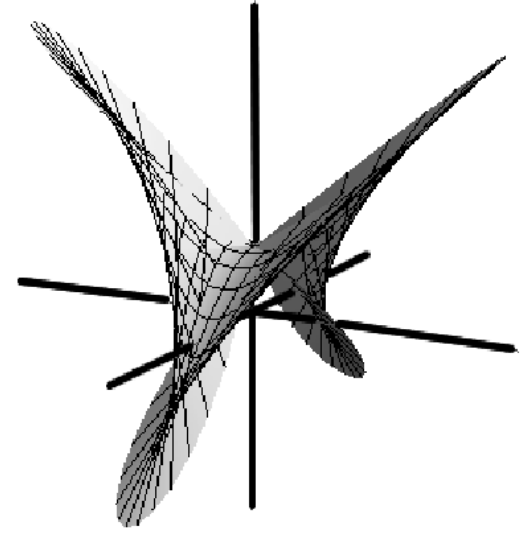

Lorentzian Enneper surface

FIGURE 1. Examples of a maxface with singularities.

is, cuspidal edges and swallowtails, appear in CMC surfaces in $\mathbb{L}^{3}$. However, the author does not know of any concrete example of CMC surfaces with swallowtails.

Recently, useful criteria for cuspidal edges and swallowtails as wave-front singularities in 3-dimensional space were given in [Kokubu et al. 05].

In this paper, we generalize the notion of CMC surfaces and construct examples with cuspidal edges and swallowtails using their criteria.

Akutagawa and Nishikawa gave a Weierstrass-type representation formula for CMC surfaces in $\mathbb{L}^{3}$ [Akutagawa and Nishikawa 90] as an analogy of the Kenmotsu formula for CMC surfaces in $\mathbb{R}^{3}$ [Kenmotsu 79]. The Gauss map $g$ of a nonsingular CMC surface is a harmonic map into the upper or lower connected component of the two-sheeted hyperboloid in $\mathbb{L}^{3}$. By stereographic projection from $(1,0,0)$ of the hyperboloid to the plane, the Gauss map $g$ can be expressed as a harmonic map into $\mathbb{C} \cup\{\infty\} \backslash\{\zeta \in \mathbb{C}|| \zeta \mid=1\}$. Making use of this result, we construct a class of CMC surfaces with singularities.

This paper is organized as follows. In Section 2, we recall some basic facts on CMC surfaces in $\mathbb{L}^{3}$. In Section 3 , we define a class of CMC surfaces with certain types of singularities, called extended CMC surfaces, by extending harmonic maps to the maps into $\mathbb{C} \cup\{\infty\}$, and we characterize the singular set. In Section 4, applying the results on singularities in [Kokubu et al. 05], we investigate singularities of a type of generalized CMC surface and give criteria for a given singular point to be $\mathcal{A}$-equivalent to a cuspidal edge, a swallowtail, or a cuspidal cross cap. In Section 5, we give concrete examples of extended CMC surfaces with cuspidal edges and swallowtails.

\section{PRELIMINARIES}

Minkowski 3-space $\mathbb{L}^{3}$ is the 3 -dimensional affine space $\mathbb{R}^{3}$ with the inner product

$$
\langle,\rangle:=-\left(d x^{0}\right)^{2}+\left(d x^{1}\right)^{2}+\left(d x^{2}\right)^{2},
$$

where $\left(x^{0}, x^{1}, x^{2}\right)$ is the standard coordinate system of $\mathbb{R}^{3}$. A vector $v \neq 0$ in $\mathbb{L}^{3}$ is called spacelike, timelike, null if $\langle v, v\rangle>0,\langle v, v\rangle\langle 0,\langle v, v\rangle=0$, respectively. An immersion $f: M^{2} \longrightarrow \mathbb{L}^{3}$ of a 2-manifold $M^{2}$ into $\mathbb{L}^{3}$ is called spacelike if the induced metric

$$
d s^{2}:=f^{*}\langle,\rangle=\langle d f, d f\rangle
$$

is positive definite on $M^{2}$.

The unit normal vector $\nu$ of a spacelike immersion $f$ is a unit timelike vector perpendicular to the tangent plane. Moreover, it can be regarded as a map

$$
\nu: M^{2} \longrightarrow \mathbb{H}^{2}=\mathbb{H}_{+}^{2} \cup \mathbb{H}_{-}^{2},
$$

where

$$
\begin{aligned}
& \mathbb{H}_{+}^{2}:=\left\{\nu=\left(\nu^{0}, \nu^{1}, \nu^{2}\right) \in \mathbb{L}^{3} \mid\langle\nu, \nu\rangle=-1, \nu^{0}>0\right\}, \\
& \mathbb{H}_{-}^{2}:=\left\{\nu=\left(\nu^{0}, \nu^{1}, \nu^{2}\right) \in \mathbb{L}^{3} \mid\langle\nu, \nu\rangle=-1, \nu^{0}<0\right\} .
\end{aligned}
$$

The map $\nu: M^{2} \longrightarrow \mathbb{H}^{2}$ is called the Gauss map of $f$. Let $\pi: \mathbb{H}^{2} \longrightarrow \mathbb{C} \cup\{\infty\}$ be the stereographic projection from the north pole $(1,0,0)$ given by

$$
\pi\left(\nu^{0}, \nu^{1}, \nu^{2}\right)=\frac{\nu^{1}+\sqrt{-1} \nu^{2}}{1-\nu^{0}} .
$$



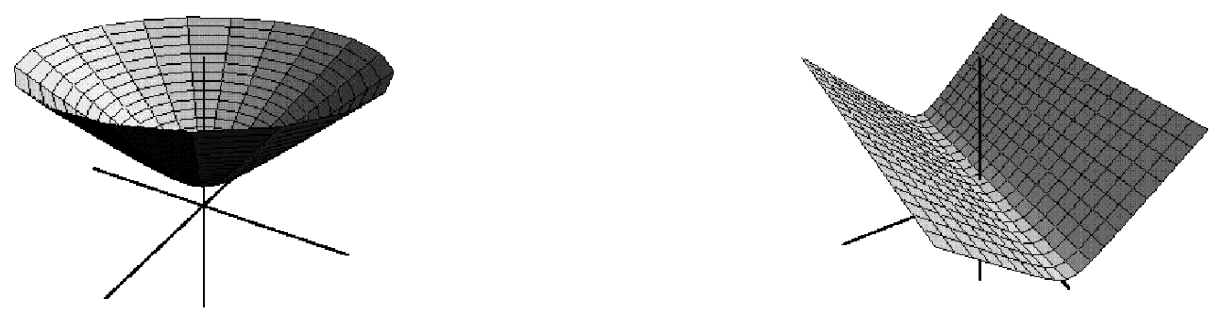

Pseudosphere

Lorentz cylinder

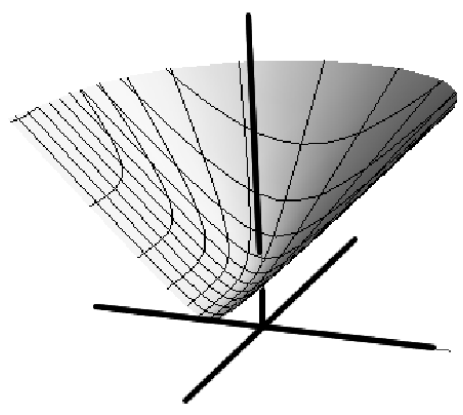

A surface of revolution about a spacelike axis

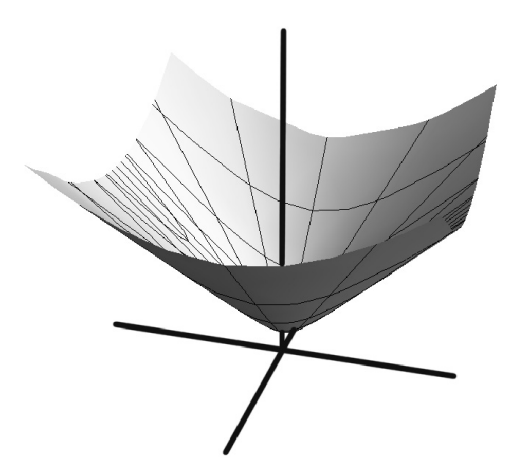

A surface of revolution about a timelike axis

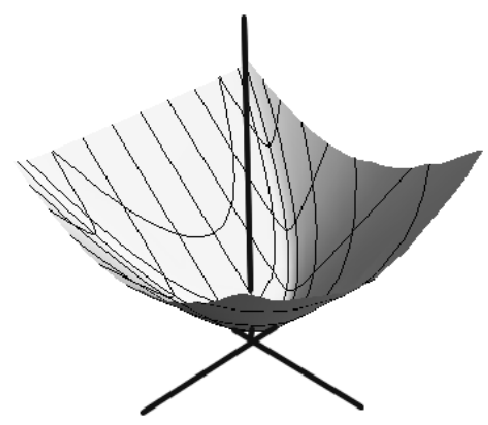

A surface of revolution about a null axis

FIGURE 2. Examples of complete CMC surfaces.

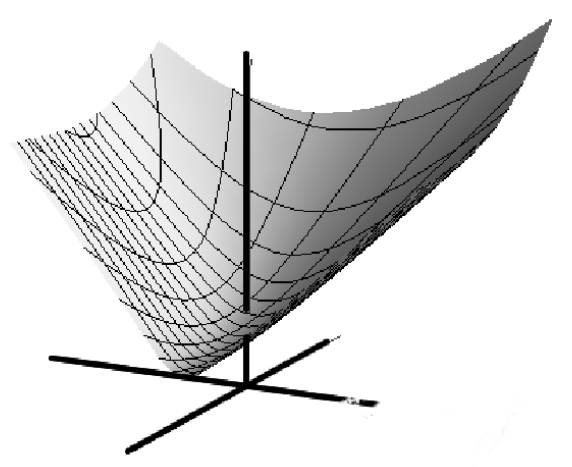

FIGURE 3. A complete surface constructed by H. Wang.

We also call the composition $g:=\pi \circ \nu$ the Gauss map of $f$. Since $\nu$ takes values in the set $\mathbb{H}^{2},|g| \neq 1$ holds on $M^{2}$.

Let $(u, v)$ be a local coordinate system of $M^{2}$. We write the first fundamental form of $f$ by

$$
d s^{2}=\langle d f, d f\rangle=E d u^{2}+2 F d u d v+G d v^{2},
$$

and the second fundamental form of $f$ by

$$
I I=\langle d f, d \nu\rangle=L d u^{2}+2 M d u d v+N d v^{2} .
$$

Then the mean curvature $H$ of $f$ is given by

$$
H=\frac{E N-2 F M+G L}{2\left(E G-F^{2}\right)} .
$$

A spacelike immersion $f: M^{2} \longrightarrow \mathbb{L}^{3}$ is said to have constant mean curvature if $H$ is equal to a nonzero constant. 
In particular, if $H=0$, the spacelike immersion is said to be maximal. A map $g: M^{2} \longrightarrow \pi\left(\mathbb{H}^{2}\right)$ defined on a Riemann surface $M^{2}$ is called harmonic if it satisfies the following condition:

$$
\frac{\partial^{2} g}{\partial z \partial \bar{z}}+\frac{2 \bar{g}}{1-|g|^{2}} \frac{\partial g}{\partial z} \frac{\partial g}{\partial \bar{z}}=0
$$

where $z$ is a complex coordinate of $M^{2}$.

It is well known that the Gauss map of a spacelike constant-mean-curvature immersion is harmonic and the Gauss map of a spacelike maximal immersion is meromorphic (see [Kobayashi 83]; see also [Kobayashi 84]). The unit normal vector field $\nu$ of the constant-mean-curvature surface in $(2-1)$ is rewritten as

$$
\nu=\frac{1}{1-|g|^{2}}\left(-\left(1+|g|^{2}\right), 2 \operatorname{Re} g, 2 \operatorname{Im} g\right) .
$$

For CMC surfaces in $\mathbb{L}^{3}$, an analogue of the Weierstrass representation formula is given in [Akutagawa and Nishikawa 90].

Theorem 2.1. [Akutagawa and Nishikawa 90] Let $M^{2}$ be a Riemann surface and $f: M^{2} \longrightarrow \mathbb{L}^{3}$ a conformal immersion with nonzero constant mean curvature $H$. Then there exists a harmonic map $g$ such that

$f=\frac{2}{H} \int_{z_{0}}^{z} \operatorname{Re}\left(\left(-2 g,\left(1+g^{2}\right), \sqrt{-1}\left(1-g^{2}\right)\right) \frac{\bar{g}_{z}}{\left(1-|g|^{2}\right)^{2}}\right) d z$,

where $z_{0} \in M^{2}$ is a base point and $g_{z}$ means $\partial g / \partial z$. Conversely, assume that $M^{2}$ is simply connected, and take a nonholomorphic harmonic map $g: M^{2} \longrightarrow \pi\left(\mathbb{H}^{2}\right)$. Then the integration in (2-3) does not depend on the choice of a path joining $z_{0}$ and $z$, and $f$ in $(2-3)$ is a spacelike immersion of constant mean curvature $H$ with Gauss map g. Furthermore, the induced metric of $f$ is given by

$$
d s^{2}=\left(\frac{2}{H\left(1-|g|^{2}\right)}\left|g_{\bar{z}}\right|\right)^{2}|d z|^{2},
$$

and the Gaussian curvature $K$ is given by

$$
K=-H^{2}\left(\left|\frac{g_{z}}{g_{\bar{z}}}\right|^{2}-1\right) .
$$

\section{CONSTANT-MEAN-CURVATURE SURFACES WITH SINGULARITIES}

In this section, we give the definition of a generalized CMC surface as a constant-mean-curvature surface with singularities. First, we review the definition of maxfaces and generalized maximal surfaces.
A holomorphic map $F=\left(F^{0}, F^{1}, F^{2}\right): M^{2} \longrightarrow \mathbb{C}^{3}$ of a Riemann surface $M^{2}$ into the complex Euclidean space $\mathbb{C}^{3}$ is called null if $\sum_{j=1}^{3} F_{z}^{j} \cdot F_{z}^{j}$ vanishes. We consider the projection

$$
\pi_{L}: \mathbb{C}^{3} \ni\left(\zeta^{1}, \zeta^{2}, \zeta^{3}\right) \longmapsto \operatorname{Re}\left(-\sqrt{-1} \zeta^{3}, \zeta^{1}, \zeta^{2}\right) \in \mathbb{L}^{3} .
$$

The projection of null holomorphic immersions into $\mathbb{L}^{3}$ by $\pi_{L}$ gives spacelike maximal surfaces with singularities, called maxfaces (see [Umehara and Yamada 06] for details). The holomorphic null immersion $F$ as above is called the holomorphic lift of the maxface.

The following fact is a generalization of the Weierstrass representation formula for a maximal surface [Kobayashi 83].

Fact 3.1. [Umehara and Yamada 06] Let $M^{2}$ be a simply connected Riemann surface and $(g, \omega)$ a pair consisting of a meromorphic function $g$ and a holomorphic 1-form $\omega$ on $M^{2}$ such that

$$
\left(1+|g|^{2}\right)^{2}|\omega|^{2} \neq 0
$$

on $U$. Then

$$
f(z):=\operatorname{Re} \int_{z_{0}}^{z}\left(-2 g, 1+g^{2}, \sqrt{-1}\left(1-g^{2}\right)\right) \omega
$$

gives a maxface in $\mathbb{L}^{3}$. Moreover, all maxfaces are locally obtained in this manner.

The induced metric by $f$ in $(3-1)$ is given by

$$
d s^{2}=\left(1-|g|^{2}\right)^{2}|\omega|^{2} .
$$

In particular, $z \in M^{2}$ is a singular point of $f$ if and only if $|g|=1$, and the restriction $f: M^{2} \backslash\{z|| g(z) \mid=1\} \longrightarrow$ $\mathbb{L}^{3}$ is a spacelike maximal immersion.

If $F: M^{2} \longrightarrow \mathbb{C}^{3}$ is null (not necessarily an immersion) and

$$
-\left|d F^{0}\right|^{2}+\left|d F^{1}\right|^{2}+\left|d F^{2}\right|^{2}
$$

does not identically vanish, then $\pi_{L} \circ F$ is a generalized maximal surface in the sense of [Estudillo and Romero 92]. The points where $F$ is not immersed are isolated branch points. In this sense, a maxface is a generalized maximal surface without branch points.

We generalize the notion of CMC surfaces in a similar way.

Definition 3.2. A smooth map $g: M^{2} \longrightarrow \mathbb{C} \cup\{\infty\}$ of a Riemann surface $M^{2}$ into the sphere $\mathbb{S}^{2}=\mathbb{C} \cup\{\infty\}$ is called a regular extended harmonic map to the hyperbolic plane if 
(1) $\omega:=\hat{\omega} d z$ can be extended to a 1 -form of class $C^{1}$ across $\left\{p \in M^{2}|| g(p) \mid=1\right\}$,

(2) $g_{z \bar{z}}+2\left(1-|g|^{2}\right) \bar{g} g_{z} \overline{\hat{\omega}}=0$ holds,

where

$$
\hat{\omega}:=\frac{\bar{g}_{z}}{\left(1-|g|^{2}\right)^{2}}
$$

and $z$ is a complex coordinate of $M^{2}$.

Remark 3.3. If $|g| \neq 1$, a regular extended harmonic map $g$ is a harmonic map.

Remark 3.4. The conjugate map $\bar{g}$ of a harmonic map $g$ is also harmonic. However, the conjugate map $\bar{g}$ of the regular extended harmonic map $g$ is not always a regular extended harmonic map, because of the condition (1). Existence of a regular extended harmonic map whose conjugate map is also a regular extended harmonic map is an open problem. This problem is related to the study of surfaces of negative constant Gaussian curvature with singularities (see [Gálvez et al. 03]).

Remark 3.5. Let $g: M^{2} \longrightarrow \mathbb{C} \cup\{\infty\}$ be a regular extended harmonic map. Then the integral $(2-3)$ is independent of the choice of path.

Definition 3.6. Let $g: M^{2} \longrightarrow \mathbb{C} \cup\{\infty\}$ be a regular extended harmonic map and $H$ a nonzero constant. Then the map $f: M^{2} \longrightarrow \mathbb{L}^{3}$ given explicitly by

$$
f=\frac{2}{H} \int_{z_{0}}^{z} \operatorname{Re}\left(-2 g, 1+g^{2}, \sqrt{-1}\left(1-g^{2}\right)\right) \omega
$$

is called a generalized constant-mean-curvature (CMC) surface with mean curvature $H$. The map $g$ is called the Gauss map.

Remark 3.7. The induced metric of the generalized CMC surface $f$ is given by

$$
d s^{2}=\left(\frac{2\left|g_{\bar{z}}\right|}{H\left(1-|g|^{2}\right)}\right)^{2}|d z|^{2}=\left(\frac{2\left(1-|g|^{2}\right)|\omega|}{H}\right)^{2} .
$$

Proposition 3.8. Let $f: M^{2} \longrightarrow \mathbb{L}^{3}$ be a generalized $C M C$ surface with Gauss map $g$. Then a point

$$
p \in M^{2} \backslash\left\{q \in M^{2}|| g(q) \mid=\infty\right\}
$$

is a singular point (that is, $\operatorname{rank} d f(p)<2$ ) if and only if $|g(p)|=1$ or $\hat{\omega}=0$. In particular, the point $p$ is a singular point with rank $d f(p)=0$ if and only if $\hat{\omega}=0$.
A point $q \in\left\{p \in M^{2}|| g(q) \mid=\infty\right\}$ is a singular point if and only if $g^{2} \hat{\omega}=0$. Moreover, such a singular point $p$ satisfies $\operatorname{rank} d f(p)=0$.

Proof: We assume that $f$ is written as in (3-2). Let $z=u+\sqrt{-1} v$ be a complex coordinate of $M^{2}$ around $p$. Without loss of generality, we may assume $H=2$. Then

$$
\begin{aligned}
& f_{z}=\frac{1}{2}\left(-2 g, 1+g^{2}, \sqrt{-1}\left(1-g^{2}\right)\right) \hat{\omega}, \\
& f_{\bar{z}}=\frac{1}{2}\left(-2 \bar{g}, 1+\bar{g}^{2},-\sqrt{-1}\left(1-\bar{g}^{2}\right)\right) \overline{\hat{\omega}} .
\end{aligned}
$$

Thus, we have

$$
\begin{aligned}
f_{u} & =\left(f_{z}+f_{\bar{z}}\right) \\
& =\left(-2 \operatorname{Re}(g \hat{\omega}), \operatorname{Re}\left(\left(1+g^{2}\right) \hat{\omega}\right),-\operatorname{Im}\left(\left(1-g^{2}\right) \hat{\omega}\right)\right), \\
f_{v} & =\sqrt{-1}\left(f_{z}-f_{\bar{z}}\right) \\
& =\left(2 \operatorname{Im}(g \hat{\omega}),-\operatorname{Im}\left(\left(1+g^{2}\right) \hat{\omega}\right),-\operatorname{Re}\left(\left(1-g^{2}\right) \hat{\omega}\right)\right), \\
f_{u} \times f_{v} & =-2 \sqrt{-1} f_{z} \times f_{\bar{z}} \\
& =\left(|g|^{2}-1\right)|\hat{\omega}|^{2}\left(1+|g|^{2}, 2 \operatorname{Re} g, 2 \operatorname{Im} g\right),
\end{aligned}
$$

where $\times$ is the Euclidean vector product in $\mathbb{R}^{3}$. These imply that $\operatorname{rank} d f(p)<2$ if and only if $|g(p)|=1$ or $\hat{\omega}(p)=0$ on $p \in M^{2} \backslash\left\{q \in M^{2}|| g(q) \mid=\infty\right\}$. Moreover, $\operatorname{rank} d f(p)=0$ if and only if $\hat{\omega}=0$. On the other hand, on $\left\{p \in M^{2}|| g(p) \mid=\infty\right\}$, we have that $\operatorname{rank} d f(p)<2$ if and only if $g^{2} \hat{\omega}=0$, and in fact, $\operatorname{rank} d f(p)=0$.

Definition 3.9. We assume that $\omega=\hat{\omega} d z$ never vanishes on $\left\{p \in M^{2}|| g(p) \mid<\infty\right\}$ and that $g^{2} \omega=g^{2} \hat{\omega} d z$ does not vanish on $\left\{p \in M^{2}|| g(p) \mid=\infty\right\}$. A regular extended harmonic map with such a property is called an extended harmonic map, and a generalized CMC surface with an extended harmonic map is called an extended $C M C$ surface. Moreover, this extended harmonic map is also called the Gauss map of the extended CMC surface.

Corollary 3.10. Let $f: M^{2} \longrightarrow \mathbb{L}^{3}$ be an extended $C M C$ surface with Gauss map $g$. Then a point $p \in U$ is a singular point if and only if $|g(p)|=1$.

\section{TYPES OF SINGULARITIES OF EXTENDED CMC SURFACES}

In the previous section, we defined generalized CMC surfaces as constant-mean-curvature surfaces with singularities. So it is quite natural to investigate which types of singularities appear on generalized CMC surfaces. In this section, we recall wave fronts in $\mathbb{R}^{3}$ and give simple criteria for a given singular point on the extended CMC surface to be each of the typical examples of singular points: 
cuspidal edges, swallowtails, and cuspidal cross caps using criteria in [Kokubu et al. 05, Fujimori et al. 08].

Let $U$ be a domain in $\mathbb{R}^{2}$ and $f: U \longrightarrow \mathbb{R}^{3}$ a $C^{\infty}$-map from $U$ into Euclidean 3 -space $\mathbb{R}^{3}$. The map $f$ is called a frontal if there exists a unit vector field $\boldsymbol{n}$ in $\mathbb{R}^{3}$ along $f$ such that $\boldsymbol{n}$ is perpendicular to $f_{*}(T U)$. We call $\boldsymbol{n}$ a unit normal vector field of a frontal $f$. We identify the unit cotangent bundle of $\mathbb{R}^{3}$ with

$$
\mathbb{R}^{3} \times S^{2}=\left\{(x, \boldsymbol{n}) \mid x \in \mathbb{R}^{3}, \boldsymbol{n} \in S^{2}\right\} .
$$

Then

$$
\begin{aligned}
& \xi:=n_{1} d x^{1}+n_{2} d x^{2}+n_{3} d x^{3}, \\
& x=\left(x^{1}, x^{2}, x^{3}\right), \quad \boldsymbol{n}=\left(n_{1}, n_{2}, n_{3}\right),
\end{aligned}
$$

gives the canonical contact form and

$$
L:=\left(f_{L}, \boldsymbol{n}\right): U \longrightarrow \mathbb{R}^{3} \times S^{2}
$$

is called a Legendrian if the pullback of the contact form $\xi$ vanishes, that is, if $\left(f_{L}\right)_{u}$ and $\left(f_{L}\right)_{v}$ are both perpendicular to $\boldsymbol{n}$, where $(u, v)$ is a local coordinate system of $U$.

In this terminology, a frontal is a projection of a Legendrian map $L$. If $L$ is a Legendrian immersion, the projection $f_{L}$ of $L$ into $\mathbb{R}^{3}$ is called a (wave) front. Now let $L=(f, \boldsymbol{n}): U \longrightarrow \mathbb{R}^{3} \times S^{2}$ be a Legendrian immersion. Then a point $p \in U$ where $f$ is not an immersion is called a singular point of the front $f$.

By definition, there exists a smooth function $\lambda$ on $U$ such that

$$
f_{u} \times f_{v}=\lambda \boldsymbol{n}
$$

where $x$ is the Euclidean vector product on $\mathbb{R}^{3}$. A singular point $p \in U$ is called nondegenerate if $d \lambda$ does not vanish at $p$.

It is well known that cuspidal edges and swallowtails are generic singularities of fronts (see [Arnol'd et al. 85, p. 336]), where a cuspidal edge and a swallowtail are respectively a singular point that is $\mathcal{A}$-equivalent at the origin to the $C^{\infty}$-map germs

$$
\begin{aligned}
& f_{C}(u, v):=\left(u^{2}, u^{3}, v\right) \\
& f_{S}(u, v):=\left(3 u^{4}+u^{2} v, 4 u^{3}+2 u v, v\right)
\end{aligned}
$$

(see Figure 4).

Here, two $C^{\infty}$-map germs $f:(U, p) \longrightarrow \mathbb{R}^{3}$ and $g:(V, q) \longrightarrow \mathbb{R}^{3}$ are $\mathcal{A}$-equivalent at the points $p \in U$ and $q \in V$ if there are a diffeomorphism germ $\varphi$ of $\mathbb{R}^{2}$ with $\varphi(p)=q$ and a local diffeomorphism $\Phi$ of $\mathbb{R}^{3}$ with $\Phi(f(p))=g(q)$ such that $g=\Phi \circ f \circ \varphi^{-1}$. A cuspidal cross cap is a singular point that is $\mathcal{A}$-equivalent to the $C^{\infty}$-map germ

$$
f_{\text {ccc }}(u, v):=\left(u^{2}, v^{2}, u v^{3}\right)
$$

at the origin (see Figure 4, right). A cuspidal cross cap is the generic singularity of frontals, but it is not a front.

Now we identify Minkowski 3 -space $\mathbb{L}^{3}$ with the 3 dimensional affine space $\mathbb{R}^{3}$. We give the following theorem as analogous to [Umehara and Yamada 06, Theorem 3.1] and [Fujimori et al. 08, Theorem 2.3].

Theorem 4.1. Let $f: M^{2} \longrightarrow \mathbb{L}^{3}$ be an extended $C M C$ surface with Gauss map $g$, and set $\hat{\omega}=\hat{g}_{z} /\left(1-|g|^{2}\right)^{2}$, where $g_{z}=\partial g / \partial z$. Then:

(1) A point $p \in U$ is a singular point if and only if $|g|=1$.

(2) $f$ is a front at a singular point $p$ if and only if

$$
\operatorname{Re}\left(\frac{g_{z}}{g^{2} \hat{\omega}}\right) \neq 0
$$

holds at $p$.

(3) $f$ is $\mathcal{A}$-equivalent to a cuspidal edge at $p$ if and only if

$$
\operatorname{Re}\left(\frac{g_{z}}{g^{2} \hat{\omega}}\right) \neq 0 \quad \text { and } \quad \operatorname{Im}\left(\frac{g_{z}}{g^{2} \hat{\omega}}\right) \neq 0
$$

hold at $p$.

(4) $f$ is $\mathcal{A}$-equivalent to a swallowtail at $p$ if and only if

$$
\frac{g_{z}}{g^{2} \hat{\omega}} \in \mathbb{R} \backslash\{0\}
$$

and

$$
\operatorname{Re}\left\{\frac{g}{g_{z}}\left(\frac{g_{z}}{g^{2} \hat{\omega}}\right)_{z}\right\} \neq \operatorname{Re}\left\{\overline{\left(\frac{g}{g_{z}}\right)}\left(\frac{g_{z}}{g^{2} \hat{\omega}}\right)_{\bar{z}}\right\}
$$

hold at $p$.

(5) $f$ is $\mathcal{A}$-equivalent to a cuspidal cross cap at $p$ if and only if

$$
\frac{g_{z}}{g^{2} \hat{\omega}} \in \sqrt{-1} \mathbb{R} \backslash\{0\}
$$

and

$$
\operatorname{Im}\left\{\frac{g}{g_{z}}\left(\frac{g_{z}}{g^{2} \hat{\omega}}\right)_{z}\right\} \neq \operatorname{Im}\left\{\overline{\left(\frac{g}{g_{z}}\right)}\left(\frac{g_{z}}{g^{2} \hat{\omega}}\right)_{\bar{z}}\right\}
$$

hold at $p$. 


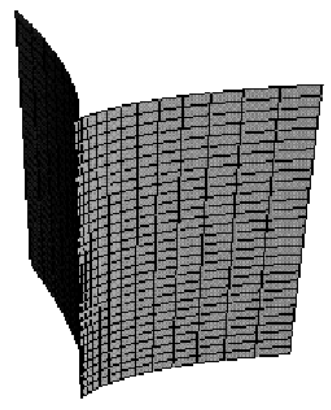

$\left(u^{2}, u^{3}, v\right)$

Cuspidal edge

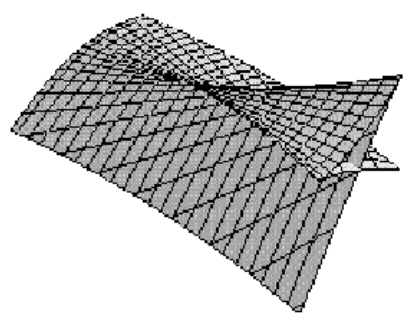

$\left(3 u^{2}+u^{2} v, 4 u^{3}+2 u v, v\right)$ Swallowtail

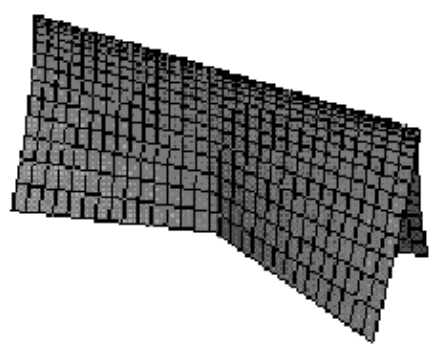

$\left(u^{2}, v^{2}, u v^{3}\right)$

Cuspidal cross cap

FIGURE 4. Examples of singularities.

We assume that $p$ is a nondegenerate singular point. Then there exists a regular curve in a neighborhood of the point $p$,

$$
\gamma=\gamma(t):(-\epsilon, \epsilon) \longrightarrow U
$$

(called the singular curve), such that $\gamma(0)=p$ and the image of $\gamma$ coincides with the set of singular points of $f$ around $p$.

On the other hand, a nonzero vector $\eta \in T U$ such that $d f(\eta)=0$ is called the null direction. For each point $\gamma(t)$, the null direction $\eta(t)$ is determined uniquely up to scalar multiplication. We review the following criteria.

Fact 4.2. [Kokubu et al. 05] Let $f: U \longrightarrow \mathbb{R}^{3}$ be a front and $p=\gamma(0) \in U$ a nondegenerate singular point of $f$.

(1) The germ of the image of the front at $p$ is $\mathcal{A}$ equivalent to a cuspidal edge if and only if $\eta(0)$ is not proportional to $\gamma^{\prime}(0)$, where ${ }^{\prime}=d / d t$.

(2) The germ of the image of the front at $p$ is $\mathcal{A}$ equivalent to a swallowtail if and only if $\eta(0)$ is proportional to $\gamma^{\prime}(0)$ and

$$
\left.\frac{d}{d t} \operatorname{det}\left(\gamma^{\prime}(t), \eta(0)\right)\right|_{t=0} \neq 0 .
$$

Fact 4.3. [Fujimori et al. 08] Let $f: U \longrightarrow \mathbb{R}^{3}$ be a frontal with unit normal vector field $\boldsymbol{n}$, and $p=\gamma(0) \in U$ a nondegenerate singular point of $f$. We set $\psi(t):=$ $\operatorname{det}\left(f_{*} \gamma^{\prime}, d \boldsymbol{n}(\eta), \boldsymbol{n}\right)$. Then the germ of the image of $f$ at $p$ is $\mathcal{A}$-equivalent to a cuspidal cross cap if and only if $\eta(0)$ is transversal to $\gamma^{\prime}(0), \psi(0)=0$, and $\psi^{\prime}(0) \neq 0$.

Proof of Theorem 4.1: Though the proofs are parallel to those in [Umehara and Yamada 06, Theorem 3.1], [Umehara and Yamada 06, Lemma 3.3], and [Fujimori et al. 08,
Theorem 2.3], we give proofs here for the reader's convenience. We have already proved (1) in Corollary 3.10. We identify $\mathbb{L}^{3}$ with Euclidean 3 -space $\mathbb{R}^{3}$. Let $f: M^{2} \longrightarrow \mathbb{L}^{3}$ be an extended CMC surface with Gauss map $g$. Without loss of generality, we may assume $H=2$. Then

$$
\boldsymbol{n}=\frac{1}{\sqrt{\left(1+|g|^{2}\right)^{2}+4|g|^{2}}}\left(1+|g|^{2}, 2 \operatorname{Re} g, 2 \operatorname{Im} g\right)
$$

is the unit normal vector field of $f$ with respect to the Euclidean metric of $\mathbb{R}^{3}$. From now on, we assume $|g(p)|=1$ and $\omega(p) \neq 0$. In the sense of [Umehara and Yamada 06],

$$
\eta=\frac{\sqrt{-1}}{g \hat{\omega}}
$$

gives the null direction at $p$. On the other hand, we have

$$
d \boldsymbol{n}(p)=\frac{\sqrt{-1}}{2 \sqrt{2}}\left(\frac{d g}{g}-\frac{d \bar{g}}{\bar{g}}\right)(0, \operatorname{Im} g,-\operatorname{Re} g) .
$$

If $d g(p)=0$, then $(f, \boldsymbol{n})$ is not an immersion at $p$, because $d \boldsymbol{n}=0$. So we may assume $d g(p) \neq 0$.

Since $g_{\bar{z}}(p)=0$ by (1) in Definition 3.2, the null direction of $d \boldsymbol{n}$ at $p$ is proportional to

$$
\mu=\overline{\left(\frac{g_{z}}{g}\right)} .
$$

Thus we have (2).

Next, the function $\lambda$ in (4-1) is calculated as

$$
\lambda=\left(|g|^{2}-1\right)|\hat{\omega}|^{2} \sqrt{\left(1+|g|^{2}\right)^{2}+4|g|^{2}} .
$$

By assumption, it is sufficient to consider the case $|g|=1$ and $\omega(p) \neq 0$. Then we can obtain that if $\operatorname{Re}\left(g_{z} /\left(g^{2} \hat{\omega}\right)\right) \neq$ 0 at $p$, then $p$ is nondegenerate. 
Assume that $\operatorname{Re}\left(g_{z} /\left(g^{2} \hat{\omega}\right)\right) \neq 0$ holds at a singular point $p$. Then $f$ is a front and $p$ is a nondegenerate singular point. Since the singular set of $f$ is characterized by $g \bar{g}=1$, the singular curve $\gamma(t)$ with $\gamma(0)=p$ satisfies $g(\gamma(t) \overline{g(\gamma(t))}=1$. Differentiating this, we get

$$
\operatorname{Re}\left(\frac{g_{z}}{g} \gamma^{\prime}\right)=0
$$

because $g_{\bar{z}}(p)=0$.

This implies that $\gamma^{\prime}$ is perpendicular to $\overline{g_{z} / g}$, that is, proportional to $\sqrt{-1} g_{z} / g$. Hence we can parameterize $\gamma$ as

$$
\gamma^{\prime}(t)=\sqrt{-1} \overline{\left(\frac{g_{z}}{g}\right)}(\gamma(t)) .
$$

Thus we have (3).

Next, we assume that $\operatorname{Im}\left(g_{z} /\left(g^{2} \hat{\omega}\right)\right)=0$ holds at the singular point $p$. In this case,

$$
\begin{aligned}
\left.\frac{d}{d t}\right|_{t=0} \operatorname{det}\left(\gamma^{\prime}, \eta\right) \\
=\operatorname{Im}\left(\left(\frac{g_{z}}{g^{2} \hat{\omega}}\right)_{z} \frac{d \gamma}{d t}+\left(\frac{g_{z}}{g^{2} \hat{\omega}}\right)_{\bar{z}} \frac{d \bar{\gamma}}{d t}\right) \\
=-\operatorname{Re}\left(\frac{g_{z}}{g^{2} \hat{\omega}}\right)_{z} \overline{\left(\frac{g_{z}}{g}\right)}+\operatorname{Re}\left(\frac{g_{z}}{g^{2} \hat{\omega}}\right)_{\bar{z}}\left(\frac{g_{z}}{g}\right) \\
=-\left|\frac{g_{z}}{g}\right|^{2} \operatorname{Re}\left[\frac{g}{g_{z}}\left(\frac{g_{z}}{g^{2} \hat{\omega}}\right)_{z}\right] \\
+\left|\frac{g_{z}}{g}\right|^{2} \operatorname{Re}\left[\overline{\left(\frac{g}{g_{z}}\right)}\left(\frac{g_{z}}{g^{2} \hat{\omega}}\right)_{\bar{z}}\right] .
\end{aligned}
$$

Thus we have (4).

On the other hand, if we assume that $f$ is a frontal (not a front), then one can compute $\psi$ as in Fact 4.3 as

$$
\psi=\operatorname{det}\left(f_{*} \gamma^{\prime}, d \boldsymbol{n}(\eta), \boldsymbol{n}\right)=\operatorname{Re}\left(\frac{g_{z}}{g^{2} \hat{\omega}}\right) \cdot \psi_{0},
$$

where $\psi_{0}$ is a smooth function on a neighborhood of $p$ such that $\psi_{0}(p) \neq 0$. Then the second and third conditions of Fact 4.3 are written as

$$
\operatorname{Re}\left(\frac{g_{z}}{g^{2} \hat{\omega}}\right)=0
$$

and

$$
\operatorname{Im}\left[\frac{g}{g_{z}}\left(\frac{g_{z}}{g^{2} \hat{\omega}}\right)_{z}\right] \neq \operatorname{Im}\left[\overline{\left(\frac{g}{g_{z}}\right)}\left(\frac{g_{z}}{g^{2} \hat{\omega}}\right)_{\bar{z}}\right] .
$$

Here we have used the relation

$$
\frac{d}{d t}=\sqrt{-1}\left[\overline{\left(\frac{g_{z}}{g}\right)} \frac{\partial}{\partial z}-\frac{g_{z}}{g} \frac{\partial}{\partial \bar{z}}\right]
$$

which comes from (4-2). Using the relation $\overline{\left(g_{z} / g\right)}=$ $\left(g / g_{z}\right) \cdot($ a real-valued function), we obtain $(5)$.

\section{EXAMPLES}

We shall introduce two examples.

Example 5.1. (A surface of revolution about a timelike axis.) A surface of revolution about a timelike line, say the $x^{0}$-axis, is a surface given by

$$
f(s, t)=\left(x^{0}(s), x^{1}(s) \cos t, x^{1}(s) \sin t\right),
$$

where $\left(x^{0}(s), x^{1}(s)\right)$ is a profile curve in the $x^{0} x^{1}$-plane. In [Ishihara and Hara 88], the authors give the profile curves of CMC surfaces. If we change the coordinate to an isothermal coordinate for $T_{3}$ in [Ishihara and Hara 88, Theorem 1], then this surface is congruent to the surface obtained from the following Gauss map: Let $M^{2}=(-\pi, \pi) \times \mathbb{R}$ and let $z=u+\sqrt{-1} v \in M^{2}$ be a complex coordinate. Then the map $g: M^{2} \longrightarrow \mathbb{C} \cup\{\infty\}$ given by

$$
g(z)=\frac{\cos u}{1+\sin u} e^{\sqrt{-1} v}
$$

is an extended harmonic map. Therefore, the map $g$ gives an example of an extended CMC surface. The set of singularities is $\{z \mid \operatorname{Re} z=0\}$ and its image is a point in $\mathbb{L}^{3}$ at which the image is tangent to the light cone (see Figure 5). Such a singularity is called a conelike singularity.

Example 5.2. (A surface of revolution about a spacelike axis.) A surface of revolution about a spacelike axis, say the $x^{1}$-axis, is a surface given by

$$
f(s, t)=\left(x^{0}(s) \cosh t, x^{1}(s), x^{0}(s) \sinh t\right),
$$

where $\left(x^{0}(s), x^{1}(s)\right)$ is a profile curve in the $x^{0} x^{1}$-plane. If we change the coordinate of $S_{3}$ in [Ishihara and Hara 88, Theorem 1], then this surface is congruent to the surface obtained from the following Gauss map: Let $M^{2}=\mathbb{C}$ and $z=u+\sqrt{-1} v \in M^{2}$ be a complex coordinate. Then the map given by

$$
g(z)=\frac{\cosh u \sinh v+\sqrt{-1} e^{u}}{\sinh u+\cosh u \cosh v}
$$

is an extended harmonic map. Therefore, the map $g$ gives an example of an extended CMC surface.

The set of singularities is $\{z \mid \operatorname{Re} z=0\}$, and its image is a conelike singularity (see Figure 6).

Remark 5.3. All incomplete CMC surfaces of revolution in $\mathbb{L}^{3}$ have only conelike singularities. Moreover, all surfaces of revolution are fronts. 


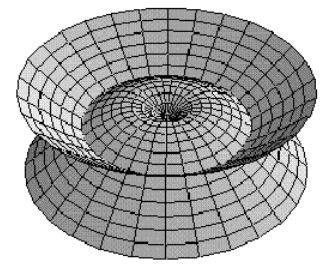

Surface of revolution about a timelike axis

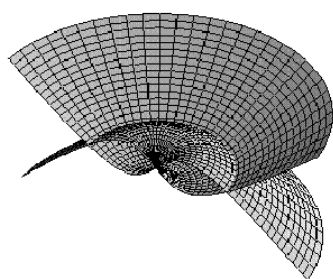

Half cut

FIGURE 5. A surface of revolution about a timelike axis.

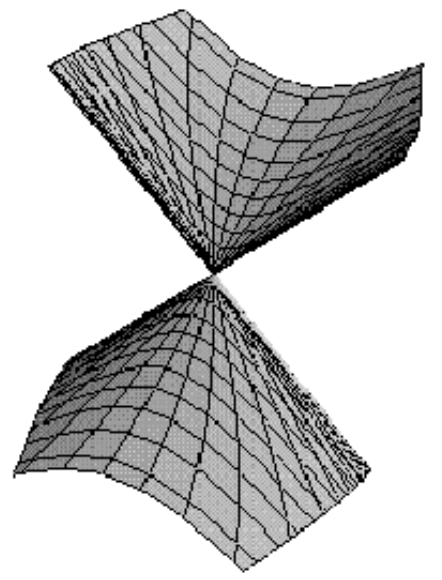

FIGURE 6. Surface of revolution about a spacelike axis.

Next, we introduce two examples with cuspidal edges and swallowtails.

\section{Example 5.4. (A helicoidal surface about a spacelike}

axis.) A helicoidal surface about a spacelike axis is the surface given by

$$
f(s, t)=\left(x^{0}(s) \cosh t, x^{1}(s)+c t, x^{0}(s) \sinh t\right),
$$

where $\left(x^{0}(s), x^{1}(s)\right)$ is a profile curve in the $x^{0} x^{1}$-plane. In [Beneki et al. 02], the authors give a method to construct helicoidal surfaces about spacelike and timelike axes with prescribed Gaussian and mean curvature. Applying their result to constant-mean-curvature surfaces, we obtain the profile curves of surfaces of constant mean curvature 1 as follows:

$$
\left(x^{0}(s), x^{1}(s)\right)=\left(s, \int \frac{\left|s^{2}+c_{1}\right| \sqrt{c^{2}-s^{2}}}{|s| \sqrt{s^{2}+\left(s^{2}+c_{1}\right)^{2}}} d s\right),
$$

where $c$ and $c_{1}$ are constants.

To consider such a surface as an extended CMC surface, it is necessary to find an isothermal coordinate of this surface. When $c_{1}=0$, this surface is congruent to the surface obtained from the following Gauss map: we set

$$
\begin{aligned}
& \xi=\sqrt{\frac{c^{2}+1+\sqrt{c^{2}+1}}{2}} u-\sqrt{\frac{c^{2}+1-\sqrt{c^{2}+1}}{2}} v, \\
& \eta=-\sqrt{\frac{c^{2}+1-\sqrt{c^{2}+1}}{2}} u+\sqrt{\frac{c^{2}+1+\sqrt{c^{2}+1}}{2}} v,
\end{aligned}
$$

where $z=u+\sqrt{-1} v$ is a complex coordinate. Then the domain of the Gauss map is $M^{2}=\left\{(u, v) \mid-K_{1}<\xi<\right.$ $K_{1}$ \}, where $K_{1}$ is the complete elliptic integral of the first kind with modulus $k_{1}=\sqrt{c^{2} /\left(c^{2}+1\right)}$, that is,

$$
K_{1}=K\left(k_{1}\right)=\int_{0}^{\pi / 2} \frac{d \varphi}{\sqrt{1-k_{1}^{2} \sin \varphi}}
$$

(see Figure 7, left).

Then the Gauss map $g: M^{2} \longrightarrow \mathbb{C} \cup\{\infty\}$ is given by

$$
\begin{aligned}
g(z) & \frac{\hat{c} \operatorname{cn} \xi \operatorname{dn} \xi-\sqrt{-1}(\hat{c} \operatorname{dn} \xi \sinh \eta+c \operatorname{sn} \xi \operatorname{cn} \xi \sinh \eta)}{\operatorname{sn} \xi+\hat{c} \operatorname{dn} \xi \cosh \eta+c \operatorname{sn} \xi \operatorname{cn} \xi \sinh \eta},
\end{aligned}
$$




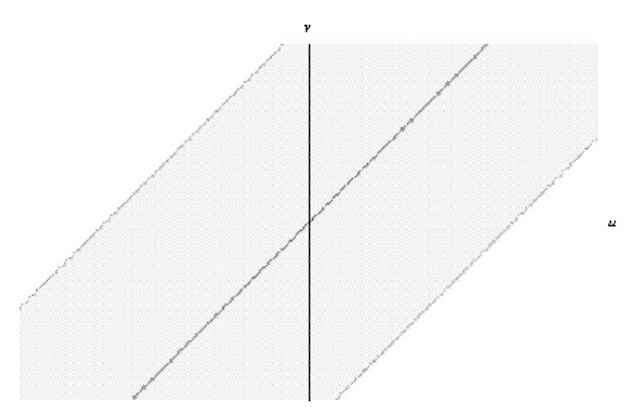

Domain of a helicoidal surface

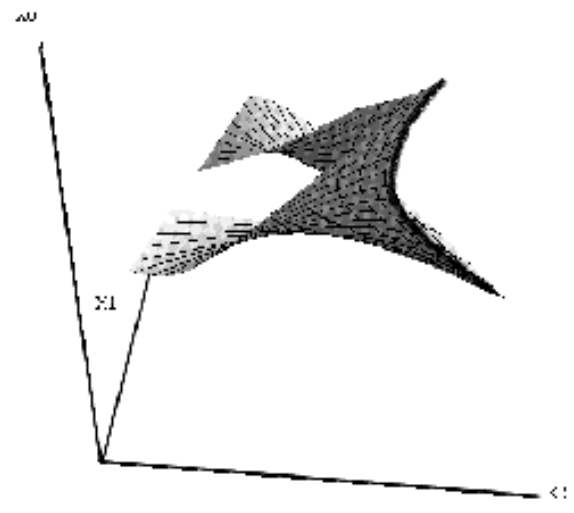

A helicoidal surface with cuspidal edge

FIGURE 7. Helicoidal surface.

where sn, cn, and dn are Jacobi's elliptic functions with modulus $k_{1}$, and to save space we have introduced the abbreviation $\hat{c}:=\sqrt{c^{2}+1}$. We get that this map is an extended harmonic map. Therefore, the map $g$ gives an example of an extended CMC surface. The set of singularities is $\{(u, v) \mid \xi(u, v)=0\}$ (see Figure 7, left). Applying this $g$ to Theorem 4.1(4), we get that the singular points are cuspidal edges. This figure is obtained when $c=2$.

Example 5.5. (Enneper-Wente-type surface.) CMC surfaces under a curvature line condition are constructed in [Abresch 87] and [Walter 87]. In this example, we construct CMC surfaces in $\mathbb{L}^{3}$ by an analogy to Walter's method. Here, the curvature line condition is the condition that each $u$-curve is to lie in some Minkowski sphere, and each $v$-curve is to lie in some plane where the Minkowski sphere is a quadric surface expressed by

$$
\left\{\left(x_{0}, x_{1}, x_{2}\right) \mid-\left(x_{0}\right)^{2}+\left(x_{1}\right)^{2}+\left(x_{2}\right)^{2}=c\right\} .
$$

When the constant $c$ is a positive number, negative number, or zero, the Minkowski sphere is respectively the hyperbolic plane $\mathbb{H}^{2}$, the de-Sitter plane $\mathbb{S}_{1}^{2}$, or the light cone.

Let $\sigma$ be a solution of the sinh-Gordon equation

$$
\frac{\partial^{2} \sigma}{\partial u^{2}}+\frac{\partial^{2} \sigma}{\partial v^{2}}=\sinh \sigma
$$

under the relation

$$
\sigma_{u v}=\frac{1}{2} \sigma_{u} \sigma_{v} \operatorname{coth} \frac{\sigma}{2}
$$

Then there is a CMC surface with the following first and second fundamental forms:

$$
\begin{aligned}
d s^{2} & =e^{\sigma}\left(d u^{2}+d v^{2}\right) \\
I I & =\frac{e^{\sigma}+1}{2} d u^{2}+\frac{e^{\sigma}-1}{2} d v^{2}
\end{aligned}
$$

with curvature line condition described above. The solution of (5-1) and (5-2) is written as

$$
\sigma=\log \left(\frac{1+k(u) l(v)}{1-k(u) l(v)}\right)^{2}
$$

with functions $k(u)$ and $l(v)$ that satisfy

$$
\begin{aligned}
2\left(k^{\prime}(u)\right)^{2} & =-p k(u)^{4}+2(1-b) k(u)^{2}+q, \\
2(\dot{l}(v))^{2} & =-q l(v)^{4}+2 b l(v)^{2}+p,
\end{aligned}
$$

where $b, p, q$ are real constants, ${ }^{\prime}=d / d u$, and ${ }^{\cdot}=d / d v$. For the sake of simplicity, we set $b=1 / 2, p=q=1$. Then we obtain solutions of $(5-3)$ and (5-4) explicitly by

$$
\begin{aligned}
& k(u)=\sqrt{\frac{1+\sqrt{5}}{2}} \text { cn }\left(\sqrt{\frac{\sqrt{5}}{2} u},\right. \\
& l(v)=\sqrt{\frac{1+\sqrt{5}}{2}} \text { cn }\left(\sqrt{\frac{\sqrt{5}}{2}} v\right),
\end{aligned}
$$

where $\mathrm{cn}$ is the Jacobi cn function of modulus

$$
\sqrt{(5+\sqrt{5}) / 10}
$$




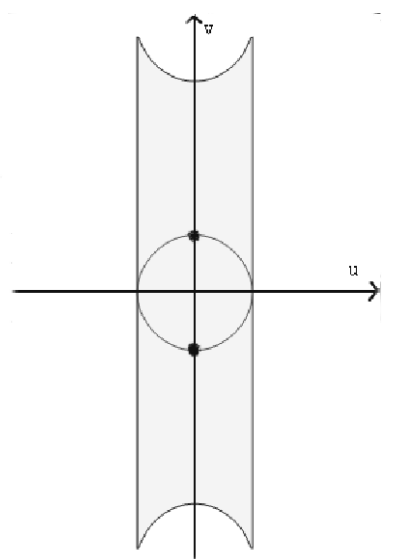

Domain of Enneper-Wente type

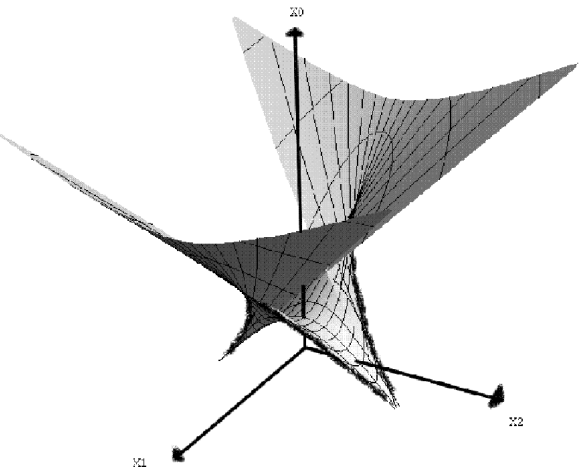

Surface with swallowtail

FIGURE 8. Enneper-Wente type surface for $b=\frac{1}{2}, p=q=1$.

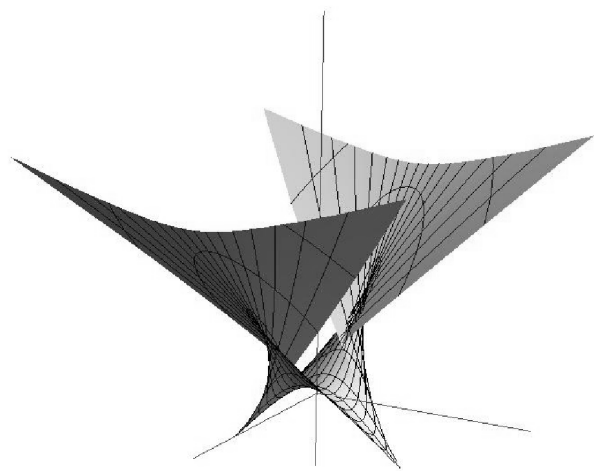

FIGURE 9. Enneper-Wente-type surface for $b=0, p=2, q=1$.

Applying Walter's construction [Walter 87] to the Minkowski case, we get a CMC surface explicitly by

$$
\begin{aligned}
& x_{0}=\frac{-h^{\prime}(u)}{\sqrt{h(u)^{2}-h^{\prime}(u)^{2}}} \sinh \mu(u)+\eta(u, v) \cosh \mu(u), \\
& x_{1}=\frac{2}{\sqrt{5}}\left(\frac{2 h(u) h^{\prime}(v)}{1-h(u) h(v)}+\int\left(-h(v)^{2}+\frac{1}{2}\right) d v\right), \\
& x_{2}=\frac{-h^{\prime}(u)}{\sqrt{h(u)^{2}-h^{\prime}(u)^{2}}} \cosh \mu(u)+\eta(u, v) \sinh \mu(u),
\end{aligned}
$$

where

$$
\begin{array}{r}
h(x)=\sqrt{\frac{1+\sqrt{5}}{2}} \operatorname{cn}\left(\sqrt{\frac{\sqrt{5}}{2}} x\right), \\
\mu(u)=\int^{u}\left(\frac{2+\left(1+\sqrt{5} h(t)^{2}\right)}{2-\left(1+\sqrt{5} h(t)^{2}\right)}\right. \\
\left.+\frac{h(t)^{4}+1}{h(t)^{4}+h(t)^{2}-1}\right) d t,
\end{array}
$$

$$
\begin{aligned}
h^{\prime}(x) & =\frac{d h}{d x}(x) \\
& =-\frac{\sqrt{5+\sqrt{5}}}{2} \operatorname{sn}\left(\sqrt{\frac{\sqrt{5}}{2}} x\right) \operatorname{dn}\left(\sqrt{\frac{\sqrt{5}}{2}} x\right), \\
\eta(u, v) & =\frac{h(u)\left(1+2 h(u)^{2}\right)-h(v)\left(2-h(u)^{2}\right)}{\sqrt{5}(1-h(u) h(v)) \sqrt{h(u)^{2}-h^{\prime}(u)^{2}}} .
\end{aligned}
$$

This surface is defined on the following domain $M^{2}$ :

$$
\begin{aligned}
M^{2}= & \left\{(u, v) \mid \operatorname{cn}\left(\sqrt{\frac{\sqrt{5}}{2}} u\right) \operatorname{cn}\left(\sqrt{\frac{\sqrt{5}}{2}} v\right)>\frac{1-\sqrt{5}}{2},\right. \\
& \left.\operatorname{cn}\left(\sqrt{\frac{\sqrt{5}}{2}} u\right)>\sqrt{\frac{3-\sqrt{5}}{2}}\right\} .
\end{aligned}
$$


Then we can calculate the Gauss map $g: M^{2} \longrightarrow \mathbb{C} \cup$ $\{\infty\}$ explicitly by

$$
\begin{aligned}
g=\frac{1}{\Delta}\{ & \left(h(u)^{3} h(v)^{2}+2 h(u)^{3}-2 h(u) h(v)^{2}\right. \\
& +h(u)) \sinh \mu(u) \\
& -\sqrt{5}\left(1-h(u)^{2} h(v)^{2}\right) h^{\prime}(u) \cosh \mu(u) \\
& \left.+4 \sqrt{-1} h(u) h^{\prime}(v) \sqrt{h(u)^{2}-h^{\prime}(u)^{2}}\right\},
\end{aligned}
$$

where

$$
\begin{aligned}
\Delta= & \sqrt{5}\left(1-h(u)^{2} h(v)^{2}\right) \sqrt{h(u)^{2}-h^{\prime}(u)^{2}} \\
& -\sqrt{5}\left(1-h(u)^{2} h(v)^{2}\right) h^{\prime}(u) \sinh \mu(u) \\
& -\left(h(u)^{3} h(v)^{2}+2 h(u)^{3}-2 h(u) h(v)^{2}\right. \\
& +h(u)) \cosh \mu(u) .
\end{aligned}
$$

The solution $\sigma$ of (5-1) diverges to $-\infty$ at some points, but the harmonic map $g$ is defined at these points smoothly. We get that this map is an extended harmonic map. Therefore, the map $g$ gives an example of an extended CMC surface. The set of singularities is

$$
\left\{(u, v) \mid \operatorname{cn}\left(\sqrt{\frac{\sqrt{5}}{2}} u\right) \operatorname{cn}\left(\sqrt{\frac{\sqrt{5}}{2}} v\right)=\frac{\sqrt{5}-1}{2}\right\}
$$

(see Figure 8, left).

By applying this $g$ to Theorem 4.1(4) and (5), we get that the points

$$
\left\{(u, v) \mid \operatorname{cn}\left(\sqrt{\frac{\sqrt{5}}{2}} u\right)=1, \text { cn }\left(\sqrt{\frac{\sqrt{5}}{2}} v\right)=\frac{\sqrt{5}-1}{2}\right\}
$$

are swallowtails, and the singular points other than the above points are cuspidal edges.

Remark 5.6. In the Euclidean case, Walter gives some doubly periodic constant-mean-curvature immersions. However, in the Minkowski case, we cannot obtain a doubly periodic constant-mean-curvature immersion by analogy to Walter's method.

Remark 5.7. Under another choice of $b, p, q$, we get a similar surface. For example, Figure 9 shows the surface when $b=0, p=2, q=1$.

Finally we conclude this paper with the following open problem.

Open Problem 5.8. Are there extended CMC surfaces in $\mathbb{L}^{3}$ with cuspidal cross cap?
It is expected that there exists such an example among Enneper-Wente-type surfaces or similar classes.

\section{ACKNOWLEDGMENTS}

The author thanks Professors Kotaro Yamada and Wayne Rossman for their useful advice. The author also thanks the referee for a careful reading of this paper and valuable comments.

\section{REFERENCES}

[Abresch 87] U. Abresch. "Constant Mean Curvature Tori in Terms of Elliptic Functions." J. reine angew. Math. 374 (1987), 169-192.

[Akutagawa 94] K. Akutagawa. "Harmonic Diffeomorphisms of the Hyperbolic Plane." Trans. Amer. Math. Soc. 342 (1994), 325-342.

[Akutagawa and Nishikawa 90] K. Akutagawa and S. Nishikawa. "The Gauss Map and Spacelike Surfaces with Prescribed Mean Curvature in Minkowski 3-Space." Tôhoku Math. J. 42 (1990), 67-82.

[Arnol'd et al. 85] V. I. Arnol'd, S. M. Gusein-Zade, and A. N. Varchenko. Singularities of Differentiable Maps, vol. 1, Monographs in Mathematics 82. Basel: Birkhäuser, 1985.

[Beneki et al. 02] C. C. Beneki, G. Kaimakamis, and B. J. Papantoniou. "Helicoidal Surfaces in Three-Dimensional Minkowski Space." J. Math. Anal. Appl. 275 (2002), 586614.

[Estudillo and Romero 92] F. J. M. Estudillo and A. Romero. "Generalized Maximal Surfaces in Lorentz-Minkowski Space $\mathbb{L}^{3}$." Math. Proc. Camb. Phil. Soc. 111 (1992), 515524.

[Fujimori et al. 08] S. Fujimori, K. Saji, M. Umehara, and K. Yamada. "Singularities of Maximal Surfaces." Math. Z. 259 (2008), 827-848.

[Gálvez et al. 03] J. A. Gálvez, A. Martínez, and F. and Milán. "Complete Constant Gaussian Curvature Surfaces in the Minkowski Space and Harmonic Diffeomorphisms onto the Hyperbolic Plane" Tôhoku Math. J. 55 (2003), 467-476.

[Hopf 51] H. Hopf. "Über Flächen mit einer Relation zwischen den Hauptkrümmungen." Math. Nachr. 4 (1951), 232249.

[Ishihara and Hara 88] T. Ishihara and F. Hara. "Surfaces of Revolution in the Lorentzian 3-Space." J. Math. Tokushima Univ. 22 (1988), 1-13.

[Kenmotsu 79] K. Kenmotsu. "Weierstrass Formula for Surfaces of Prescribed Mean Curvature." Math. Ann. 245 (1979), 89-99.

[Kobayashi 83] O. Kobayashi. "Maximal Surfaces in the 3Dimensional Minkowski Space $L^{3}$." Tokyo J. Math. 6 (1983), 297-309. 
[Kobayashi 84] O. Kobayashi. "Maximal Surfaces with Conelike Singularities." J. Math. Soc. Japan 36 (1984), 609-617.

[Kokubu et al. 05] M. Kokubu, W. Rossman, K. Saji, M. Umehara, and K. Yamada. "Singularities of Flat Fronts in Hyperbolic 3-Space." Pacific J. Math. 221 (2005), 303-351.

[Umehara and Yamada 06] M. Umehara and K. Yamada. "Maximal Surfaces with Singularities in Minkowski Space." Hokkaido Math. J. 35 (2006), 13-40.
[Walter 87] R. Walter. "Explicit Examples to the H-Problem of Heinz Hopf." Geom. Dedicata 23 (1987), 187-213.

[Wang 91] H. Wang. "The Construction of Surfaces of Constant Mean Curvature in Minkowski 3-Space." In Differential Geometry (Shanghai, 1991), pp. 243-246. River Edge, NJ: World Sci. Publ., 1993.

[Wente 86] H. C. Wente. "Counterexample to a Conjecture of H. Hopf." Pacific J. Math. 121 (1986), 193-243.

Yuhei Umeda, Graduate School of Mathematics, Kyushu University, Higashi-ku, Fukuoka, Japan

(y-umeda@math.kyushu-u.ac.jp)

Received July 8, 2008; accepted in revised form January 14, 2009. 